\title{
Dual Drug-Loaded Coaxial Nanofibers for the Treatment of Corneal Abrasion
}

\author{
Essam A. Tawfik ${ }^{1,2}$, Duncan Q.M. Craig ${ }^{1 *}$, Susan A. Barker ${ }^{1,3}$ \\ ${ }^{1}$ UCL School of Pharmacy, University College London, 29-39 Brunswick Square, \\ London WC1N 1AX, United Kingdom \\ ${ }^{2}$ National Center for Pharmaceutical Technology, King Abdulaziz City for Science \\ and Technology, 6086 Riyadh, 11442 Kingdom of Saudi Arabia \\ ${ }^{3}$ Current address: Medway School of Pharmacy, The Universities of Greenwich and \\ Kent at Medway, Anson Building, Central Avenue, Chatham, Kent ME4 4TB, United \\ Kingdom
}

*Author for correspondence; e-mail: duncan.craig@ucl.ac.uk, +44 2075819

\begin{abstract}
Corneal abrasion is a scratch wound on the surface of the anterior segment of the eye, which can predispose a patient to corneal infection and scarring, particularly if the cut penetrates to the deep corneal layers. Here we investigate a novel approach to co-administer an anti-scarring agent and an antibiotic, both being incorporated into one dosage form so as to accelerate wound closure and to treat any associated infection. More specifically, we have used electrospun fibers as a means of incorporating the two drugs into distinct compartments via coaxial electrospinning. Samples were characterised using a range of imaging, spectroscopic and thermal methods, while an HPLC assay has been developed to allow measurement of the concentration of both drug components in both the initial fibers and on release. Fibers loaded with pirfenidone in the hydrophobic polymer, PLGA, as the outer layer and moxifloxacin in the hydrophilic polymer PVP as the inner layer were successfully prepared, with smooth and non-porous surfaces and a mean diameter of circa 630 $\mathrm{nm}$. TEM image demonstrated clear distinctive layers (a core and a shell), suggesting the successful preparation of the drug-loaded coaxial fibers, supported by HPLC entrapment studies, while fluorescence microscopy confirmed the presence of the moxifloxacin within the fibers. The fibers were capable of extending the release of both drugs, hence raising the possibility of a single daily dose of the drug-loaded coaxial fibers for the treatment of corneal abrasion.
\end{abstract}

\section{Graphical abstract}

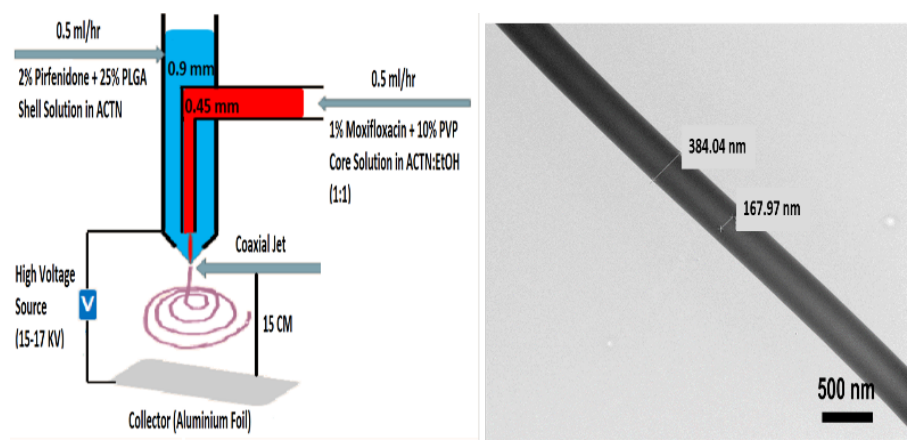




\title{
Keywords
}

Corneal abrasion, coaxial electrospinning, coaxial nanofibers, electrospun nanofibers.

\begin{abstract}
Abbreviations
ACTN, acetonitrile; $r^{2}$, coefficient of determination; DSC, Differential scanning calorimetry; DL, drug loading; EE\%, encapsulation efficiency; ECM, Extracellular matrix; FTIR, Fourier-Transform Infrared Spectroscopy; HPLC, High Performance Liquid Chromatography; PBS, Phosphate buffer saline; k, release constant; SEM, Scanning Electron Microscopy; SD, standard deviation; TGA, Thermogravimetric Analysis; TEM, Transmission Electron Microscopy; XRD, X-Ray Diffraction; Y, Yield.
\end{abstract}

\section{Introduction}

Corneal abrasion can be defined as a scratch on the surface of the eye caused by a foreign body, potentially resulting in patients suffering pain, redness, itching and blurred vision in the short term (Shahid and Harrison, 2013). More seriously, the wound may result in infection and fibrosis which may lead to significant visual loss if not treated effectively (Cheng et al., 1999, Bourcier et al., 2003; Wong et al., 2003). Indeed, opportunistic bacteria such as Pseudomonas aeruginosa ( $P$. aeruginosa), can cause corneal ulceration (erosion of the cornea) (Ong and Corbett, 2015, Stapleton and Carnt, 2012; Keay et al., 2006), while corneal scarring (corneal fibrosis) may also occur, a condition whereby epithelial cells may proliferate to form new tissue following abrasion (Kinoshita et al., 2001, Wilson et al., 1999; Salomao et al., 2011). Consequently, an immediate and effective treatment of corneal abrasion is necessary to avoid more severe complications, particularly to prevent both infection and fibrosis.

Moxifloxacin is a fourth generation fluoroquinolone antibiotic that has a high potency and low bacterial resistance compared to the older generation fluoroquinolones (Miller, 2008). This is due to its high affinity to two bacterial enzymes, DNA gyrase and topoisomerase IV (Miller, 2008; Wise, 1999). The current treatment of a corneal abrasion typically involves the use of this antibiotic as eye drops $(0.5 \% \mathrm{w} / \mathrm{v})$ three times daily for two weeks. This regiment can be unsatisfactory for many patients (Shahid and Harrison, 2013, Keay et al., 2006, Jeng and McLeod, 2003), not least due to the multiple dosing and the extensive drug loss into the tear ducts. Moreover, as a consequence of an injury, the activation of myofibroblasts and the release of fibrotic markers such as TGF- $\beta$ and TNF $\alpha$ may trigger generation of extracellular matrix (ECM) elements such as collagen, fibronectin and smooth muscle actin, leading to the proliferation of epithelial cell and the formation of a scar (Bukowiecki et al., 2017; Ljubimov and Saghizadeh, 2015). Pirfenidone is an anti-fibrotic, antiinflammatory and anti-oxidant drug that has been approved for the treatment of idiopathic pulmonary fibrosis (Raghu et al., 2011, Du Bois, 2010, lyer et al., 1999, European Medicines Agency, 2010). The drug has been reported to be, but not licensed as, an effective ocular anti-scarring treatment when used as $0.5 \% \mathrm{w} / \mathrm{v}$ eye drops (Lin et al., 2009, Zhong et al., 2011, Sun et al., 2011, Lee et al., 2014, Jung et al., 2012). However, it is recommended to be applied six times daily due to its short half-life in the ocular tissues, which has been reported to be 18 to 72 minutes (Zhong et al., 2011, Sun et al., 2011). 
We therefore propose that a dosage form involving a single daily dose of a polymeric delivery system that contains the broad-spectrum antibiotic, moxifloxacin, combined with an anti-scarring agent, pirfenidone, may accelerate the healing of the abrasion, prevent infection, reduce the development of scarring and enhance the overall patient compliance. This dual therapy, with an antibiotic and an anti-fibrotic agent, would be a sensible therapeutic approach owing to the prolonged residence time on the eye and the reduced dose frequency that can be offered by using the polymeric delivery system.

We further suggest that such a system may comprise coaxial fibers, generated by electrospinning, which could subsequently be incorporated into an application such as a sub-conjunctival insert to allow pain-free and convenient self-administration by the patient. Electrospinning is a technique used to produce micro- and nano-fibers by the application of a high voltage across a droplet of a viscous polymer solution so as to overcome the surface tension, leading to solvent evaporation and the production of a fibrous mat (Williams et al., 2018). Single- or double-layered fibers may be prepared by the use of a mono- or coaxial nozzle system, respectively. The latter may allow the incorporation of more than one material including drugs, proteins, nucleic acids and biological cells, with the ability to tailor the release rate of each loaded drug by varying the polymers used (Chakraborty et al., 2009). These fibers have been recognised for their applications in drug delivery, including wound healing, antibacterial dressing and tissue regeneration fields. This is due to their relative ease of fabrication, large surface area, tensile strength, flexibility and compatibility with biological tissues (Garg et al., 2015). In addition, the unique morphology of these fibers can potentially accelerate the wound closure by acting as a scaffold via the resemblance to tissue ECM, hence promoting cell migration and proliferation (Murugan and Ramakrishna, 2007).

It is therefore suggested that a combination of the antibiotic moxifloxacin and the antiscarring agent pirfenidone, administered by the ocular route in a combined but compartmentalized (coaxial) nanofiber formulation, may result in improved patient outcomes for corneal diseases. The use of such nanofibers as a delivery platform should inhibit any existing bacteria and promote cell proliferation and migration. For the coaxial fiber fabrication, the PLGA is designed to be the outer layer, loaded with pirfenidone, while PVP, loaded with moxifloxacin, forms the inner core. The intention is for the outer PLGA layer to allow sustained release of the drugs while also preventing burst release of the inner moxifloxacin, with both layers reflecting environments in which the incorporation of both drugs may be maximized. The stretch objective is for the compartmentalized system to allow release of both drugs in such a manner that once daily dosing becomes feasible, hence the intention in the present study is to demonstrate proof of concept that such an approach is possible.

\section{Materials and Methods}

\subsection{Materials}

A high MW PLGA 50:50 grade Purasorb® PDLG 5010 (153 kg/mol) was obtained from Corbion (Purac Biomaterials, Gorinchem, Netherlands). High MW PVP $(1,000,000-1,500,000)$ Kollidon $\AA 90 \mathrm{~F}$ was kindly donated by BASF (Germany). Ethanol, HPLC grades of water and acetonitrile (ACTN), PBS tablets with a pH 7.4, were all obtained from Sigma Aldrich Company Ltd (Sigma Aldrich, Dorset, UK). Pirfenidone was purchased from Tokyo Chemical Industry UK Ltd (The Magdalen 
Centre, Oxford, UK), while moxifloxacin hydrochloride was purchased from Cambridge Bioscience Ltd (Munro House, Cambridge, UK). Distilled water was generated by an ELGA Option 4 Water Purifier (Veolia Water Technologies, High Wycombe, UK).

\subsection{Methods}

\subsubsection{Preparation of coaxial fibers}

Coaxial fibers have been prepared by modifying the procedure described by He et al. (2018), Zhu et al. (2015) and Sun et al. (2006) using a Spraybase $®$ electrospinning instrument (Spraybase $\AA$, Dublin 2, Ireland). 25\% w/v PLGA with 2\% w/v pirfenidone was dissolved in ACTN as the outer layer (shell), while $10 \% \mathrm{w} / \mathrm{v}$ PVP and $1 \% \mathrm{w} / \mathrm{v}$ moxifloxacin was dissolved in a solvent mix of ethanol and ACTN (1:1) as the inner layer (core). A coaxial needle was used with an inner diameter of $0.45 \mathrm{~mm}$ and an outer diameter of $0.9 \mathrm{~mm}$. The flow rate for both the inner and outer layers was kept constant at $0.5 \mathrm{~mL} / \mathrm{h}$, respectively, with an applied voltage of $19 \mathrm{kV}$ and $22 \mathrm{kV}$ for the blank and drug-loaded polymeric solutions, respectively. The end-product fibrous mats were collected on aluminum foil at a tip-to-collector distance of $15 \mathrm{~cm}$. The fibers were fabricated under conditions of ambient temperature $\left(20-25^{\circ} \mathrm{C}\right)$ and relative humidity (30-45\%).

\subsubsection{Morphological characterization of the coaxial fibers}

\subsubsection{Scanning Electron Microscopy}

A $0.5 \mathrm{~cm} \times 0.5 \mathrm{~cm}$ piece of foil, on which the fibers were collected, was adhered onto an SEM stub, using double sided carbon tabs (Agar Scientific, Stansted, UK). The prepared stub was then given a thin coating of gold $(10 \mathrm{~nm})$ using a Quorum Q150T Sputter Coater (Quorum Technologies Ltd. East Sussex, UK) in an argon atmosphere. The coated stub was then transferred and imaged under FEl Quanta 200F (FEI company Ltd, Eindhoven, The Netherlands), at an acceleration voltage of $5 \mathrm{kV}$. Fiber size analysis was performed by measuring the diameter of these fibers using ImageJ software (National Institute of Health, Maryland, USA).

\subsubsection{Transmission Electron Microscopy}

Fibers were collected on a copper grid directly during fabrication. The prepared grids were stained with $2 \%$ aqueous uranyl acetate solution prior to imaging under a FEI CM120 BioTwin TEM (FEI Company Ltd. Eindhoven, The Netherlands) at an accelerating voltage of $120 \mathrm{kV}$.

\subsubsection{Light and fluorescence microscopy}

Fibers were assessed under both light and fluorescence microscopy using a Nikon Microphot-FXA microscope (Nikon, Japan). Fibers were collected directly on microscope slides and examined using total magnifications of 40X, 100X and 400X. The fluorescence wavelength used was in the range of $340 \mathrm{~nm}$.

\subsubsection{Thermal analysis and physical state characterization}

\subsubsection{Thermogravimetric Analysis}


TGA was performed using a TA Discovery thermogravimetric analyzer (TA Instruments New Castle, DE, USA). An aliquot sample (weight range 5 to $10 \mathrm{mg}$ ) was placed into an open aluminum pan. The samples were equilibrated at $30^{\circ} \mathrm{C}$ and heated to $400^{\circ} \mathrm{C}$ at a rate of $10^{\circ} \mathrm{C} /$ minute.

\subsubsection{Differential Scanning Calorimetry}

Modulated temperature DSC (MTDSC) was performed using the TA Q2000 instrument. An aliquot of each sample (weight range 3 to $5 \mathrm{mg}$ ) was weighed into a $40 \mu \mathrm{L}$ aluminum DSC pan (Perkin-Elmer) which was then sealed by an aluminum lid. Three holes have been punched through the lid after sealing. The underlying heating rate was adjusted to $2^{\circ} \mathrm{C} /$ minute with modulation parameters of amplitude $\pm 0.212^{\circ} \mathrm{C}$ and a period 40 seconds. The samples were equilibrated at $0^{\circ} \mathrm{C}$ and heated to $250^{\circ} \mathrm{C}$.

\subsubsection{Fourier-Transform Infrared Spectroscopy}

This solid state characterization was performed using Spectrum 100 FTIR spectrometer (Perkin Elmer, Massachusetts, USA). Measurements were performed with a resolution of $4 \mathrm{~cm}^{-1}$ and a duration of 16 scans over a range of $4000-650 \mathrm{~cm}^{-1}$ at ambient temperature. Spectra were plotted and analyzed using OriginPro 2016 (OriginLab Corporation, Northampton, MA, USA).

\subsubsection{X-Ray Diffraction}

Further solid state characterization was performed using a MiniFlex 600 benchtop diffractometer (RigaKu, Tokyo, Japan). The samples were placed directly on the aluminum trays. A Cu Ka radiation point source $(\lambda=1.5148227 \AA)$ was operated at 40 $\mathrm{mV}$ and $15 \mathrm{~mA}$. XRD patterns were recorded using diffraction angle $(2 \theta)$ from $3^{\circ}$ to $40^{\circ}$ (step size of $0.05^{\circ}$ and time per step of 0.2 second). Data was plotted and analyzed using OriginPro 2016 (OriginLab Corporation, Northampton, MA, USA).

\subsubsection{HPLC assay methods for determination of pirfenidone and moxifloxacin in the electrospun coaxial fibers}

The development of high performance liquid chromatography (HPLC) methods was necessary in order to measure the drug loading (DL), encapsulation efficiency (EE\%) and the release of both moxifloxacin and pirfenidone in the electrospun fibers. The HPLC system consisted of an Agilent model which contains Agilent Technologies 1200 G1329A ALS autosampler, G1322A degasser, G1311A quat pump, G1316A diode array detector, and Agilent ChemStation for LC and LC/MS systems software. Quantification was achieved by isocratic elution. These assays have been developed by combining the parameters described in Parmar et al. (2014), Dewani et al. (2011) and Ravisankar et al. (2014). An acidic HPLC grade water ( $1 \mathrm{~L}$ adjusted to $\mathrm{pH} 3.3$ by 4-5 drops of phosphoric acid) and ACTN were used as the mobile phase in a ratio of 70:30 (for the samples dissolved in ACTN) or 65:35 (for the samples dissolved in PBS) and delivered at a flow rate of $1 \mathrm{~mL} /$ minute at ambient temperature. A ZORBAX Eclipse plus C18 analytical column (C18, $5 \mu \mathrm{m}, 4.6 \mathrm{~mm} \times 250 \mathrm{~mm})$ was used. The injection volume was $20 \mu \mathrm{L}$ (for the samples dissolved in ACTN) and $50 \mu \mathrm{L}$ (for the samples dissolved in PBS). UV detection was performed at a wavelength of $310 \mathrm{~nm}$. For standard curve preparation of the samples dissolved in ACTN, serial dilutions were performed to obtain solutions of $37.5,18.75,9.375,4.688,2.344,1.172,0.586$, $0.293,0.147 \mu \mathrm{g} / \mathrm{mL}$ of each of the two drugs. For the samples dissolved in PBS, serial dilutions were performed to obtain solutions of $100,50,25,12.5,6.25,3.125,1.563$, $0.78,0.39 \mu \mathrm{g} / \mathrm{mL}$ of each of the two drugs. 


\subsubsection{Swelling and weight loss behavior}

The swelling and weight loss properties of the drug-loaded coaxial fibers were assessed by adding a defined amount of the fibers to the release medium (PBS, pH 7.4 ) in a sealed glass container with $30 \mathrm{~mL}$ of PBS and incubated at $34.3^{\circ} \mathrm{C}$. After 2 , 4,6 , and 24 hours, the wetted fiber-mat of each formulation was weighed. The percentage of swelling was calculated using the following equation:

$$
\begin{array}{ll}
\text { Swelling } \%=\frac{W-W d}{W d} \times 100 & \text { Equation } 1
\end{array}
$$

where $\mathrm{W}$ is the weight of wetted fibers and $\mathrm{Wd}$ is the dry weight (i.e. after drying) of the fibers of each formulation.

The percentage of weight loss was measured after drying the fibers in $34.3^{\circ} \mathrm{C}$ for 1 day and then reweighed to determine the dry weight using the following equation:

Weight loss $\%=\frac{W i-W d}{W i} \times 100$

Equation 2

where $\mathrm{Wi}$ is the initial weight of the fibers and $\mathrm{Wd}$ is the dried weight of the fibers of each formulation. The results represent the mean $( \pm S D)$ of at least 3 replicates.

\subsubsection{Drug loading, entrapment efficiency and yield}

For direct determination of the entrapment efficiency of loaded fibers, a certain volume of ACTN was added to a known amount of the formulation until complete dissolution. Then $1 \mathrm{~mL}$ of the formed solution is withdrawn and centrifuged for 5 minutes at 10,000 rpm at room temperature. The drug content was determined by the developed ACTN extraction HPLC method and the drug loading was calculated using the following theoretical equation:

$D L=\frac{\text { Entrapped drug amount }}{\text { Yield of fibres amount }}$

Equation 3

The entrapment efficiency was calculated by the following equation:

$E E \%=\frac{\text { Actual drug amount }}{\text { Theoretical drug amount }} \times 100$

Equation 4

While the yield $(Y)$ of the fabricated fibers was calculated by the following equation:

$Y \%=\frac{\text { Actual amount of fibres }}{\text { Theoretical amount of fibres }} \times 100$

Equation 5

where the theoretical amount of fibers was calculated by estimating the amount of the solid materials polymer \pm drug in the total volume that was injected in the electrospinning system. The results represent the mean $( \pm S D)$ of at least 3 replicates.

\subsubsection{Drug release profile}

For the release determination of the drug-loaded fibers, modified dialysis methods were used under sink condition (i.e. volume of dissolution medium which is at least 310 times the saturation volume). A known amount of the fibers was kept in custommade cages inside a sealed glass container to avoid the fibers from floating. $30 \mathrm{~mL}$ of preheated PBS, with a $\mathrm{pH} 7.4$, was added to the glass container holding the fibers inside a shaking incubator with the temperature kept constant at $34.3 \pm 0.2{ }^{\circ} \mathrm{C}$ and a shaking speed of $75 \mathrm{rpm}$ in order to mimic the corneal conditions (Efron et al., 1989, 
Garg et al., 2014, Hilal et al., 2016, Sun et al., 2016; Mirzaeei et al., 2018). After a designated time point, $1 \mathrm{~mL}$ of PBS was withdrawn and centrifuged for 5 minutes at $10,000 \mathrm{rpm}$ at room temperature, replaced by $1 \mathrm{~mL}$ of fresh buffer. The drug release content was calculated by the developed PBS extraction HPLC method and the cumulative release $\%$ was calculated using the following equation:

Cumulative release $\%=\frac{\text { Cumulative drug amount }}{\text { Theoretical drug amount }} \times 100$

Equation 6

where the cumulative drug amount is the total amount of drug released at each time point, while the theoretical drug amount is the actual amount of drug-loaded in each formulation. The results represent the mean $( \pm S D)$ of at least 3 replicates.

\section{Results and Discussion}

\subsection{Microscopy and fiber size analysis}

The SEM results illustrated that both the blank and drug-loaded coaxial fibers had smooth, un-beaded and non-porous surfaces, with no clear drug crystals present, as shown in Figure 1. These morphological features are considered to represent successful fiber preparatory criteria due to the absence of pores and roughness that can accelerate the rate of drug release profile (Williams et al., 2018).
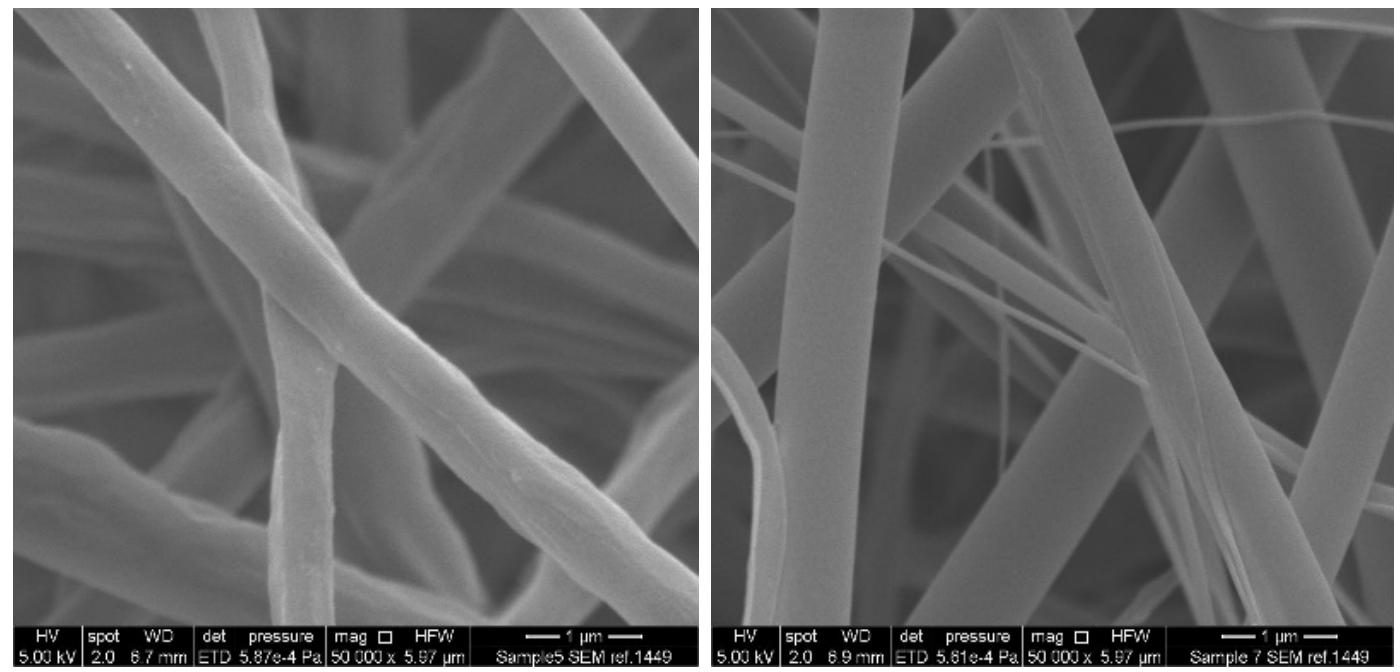

Figure 1: Surface morphology of blank (left) and drug-loaded (right) PLGA/PVP coaxial fibers.

Fiber diameters were assessed for circa 100 fibers and the mean diameters along with the standard deviations (SDs) were measured using ImageJ software. The blank coaxial fibers had a diameter of $640 \pm 220 \mathrm{~nm}$, while the diameter of the drug-loaded fibers were $630 \pm 300 \mathrm{~nm}$. Despite the addition of drugs in the drug-loaded coaxial fibers, the diameter was almost similar lower. This was due to the increase in the applied voltage that was used to overcome the higher polymer solution conductivity and viscosity that was achieved by the addition of the drugs, particularly the moxifloxacin hydrochloride. It was reported that enhancing the applied voltage would yield narrow fibers (Williams et al., 2018). The drug-loaded coaxial fibers were also observed by TEM. The fiber image in Figure 2 exhibited two clear distinctive layers that represent the inner (PVP) and outer (PLGA) layers of the coaxial fiber. This was also seen in the TEM image of He et al. (2018) PVP/PLGA (core/shell) fibers. 


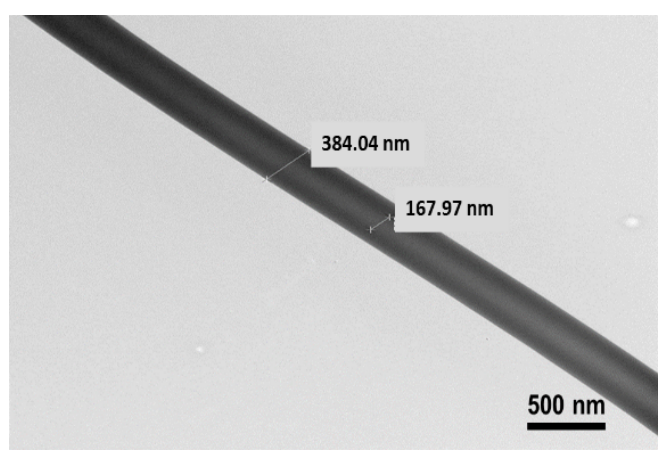

Figure 2: TEM image of the drug-loaded PLGA/PVP fibers showing the inner layer of the coaxial fibers.

Light and fluorescent microscopies were used to determine both fiber architecture (particularly the absence of beads) and to confirm the presence of the moxifloxacin in the core of these fibers, which has excitation and emission wavelengths at 290 and $500 \mathrm{~nm}$, respectively (Chan et al., 2006; Wang et al., 2016). The data is shown in Figure 3, indicating the presence of moxifloxacin within the PVP core of the coaxial fibers. Zhi-Hua et al. (2012) observed fluorescence for PVP fibers following encapsulation of fluorescein, while Tiwari et al. (2010), Zhang et al. (2006) and Romano et al. (2016) also used this approach to demonstrate encapsulation of rose bengal, fluorescently-labelled BSA and rhodamine $B$ isothiocyanate in the core of PVA/PLGA, PCL/PEG and PEO/PCL (core/shell) coaxial fibers, respectively.
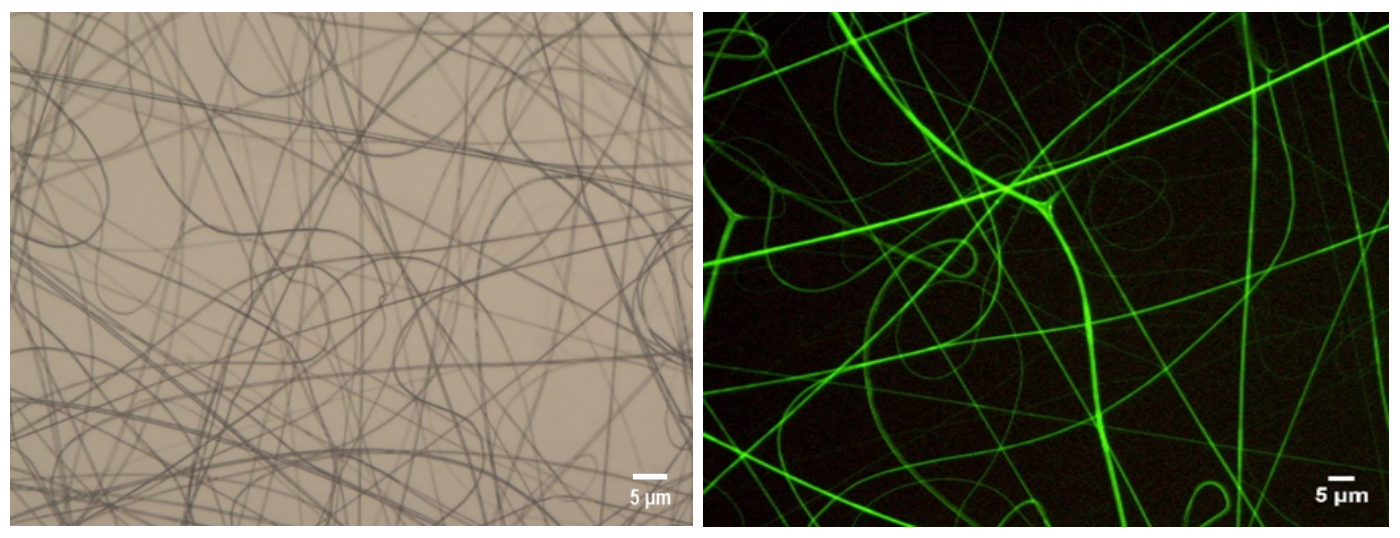

Figure 3: Light (left) and fluorescent (right) microscopic images of the drug-loaded coaxial fibers showing the absence of beaded fibers. The presence of moxifloxacin in the core of the drug-loaded coaxial fibers allowed the fibers to fluoresce.

\subsection{Thermal analysis}

Thermogravimetric analysis indicated that blank and drug-loaded coaxial fibers demonstrated a weight loss of $2 \% \pm 0.5 \%$ at $45-47^{\circ} \mathrm{C}$ which corresponded to entrapped solvent loss. A degradation onset was seen at 332 and $318^{\circ} \mathrm{C}$ for the blank and drug-loaded coaxial fibers suggesting the degradation of PLGA. A third weight loss was observed at about $368^{\circ} \mathrm{C}$ and $354^{\circ} \mathrm{C}$, indicating the degradation of PVP in the blank and drug-loaded coaxial fibers, respectively.

The MTDSC of PLGA and PVP physical mixture (PM), indicative of the unloaded coaxial fibers (Figure 4a), demonstrated two glass transition $(\mathrm{Tg})$ temperatures at $45.6^{\circ} \mathrm{C}$ and $175.7^{\circ} \mathrm{C}$ corresponding to the PLGA and PVP, respectively. The presence 
of both polymers' Tgs confirm their immiscibility on heating (Coleman and Craig, 1996). The PM of PLGA-pirfenidone/PVP-moxifloxacin that represent the drug-loaded coaxial fibers (Figure 4b), showed clear endothermic peaks of pirfenidone $\left(105.3^{\circ} \mathrm{C}\right)$ and moxifloxacin $\left(237.4^{\circ} \mathrm{C}\right)$, representing their melting temperatures $(\mathrm{Tm})$. In addition, the Tg of PLGA $\left(47.4^{\circ} \mathrm{C}\right)$ and PVP $\left(168^{\circ} \mathrm{C}\right)$ are indicated; it should be noted that the presence of sorbed water or residual solvent following processing could cause variability in the $\mathrm{Tg}$ results via plasticization effects (Jamarani et al., 2018), hence these values should not be considered immutable. Nevertheless, all these temperature points were close to those of the raw materials, with the minor differences being ascribed to the presence of the other components. It may therefore be concluded that the materials used did not significantly interact during heating, hence any changes in thermal events seen on generation of the fibers may be ascribed to changes in the physical state of the components following processing.

The MTDSC data for the blank and drug-loaded coaxial fibers (Figure 4c and $d$, respectively), showed a shift in the Tg of both PLGA and PVP polymers, with the lack of any melting peaks of the drugs. This may be ascribed to the molecular dispersion of both drugs; Zhang et al. (2012) recorded a similar observation for captopril loaded into PLGA, PLA or PCL fibrous systems.

(a)

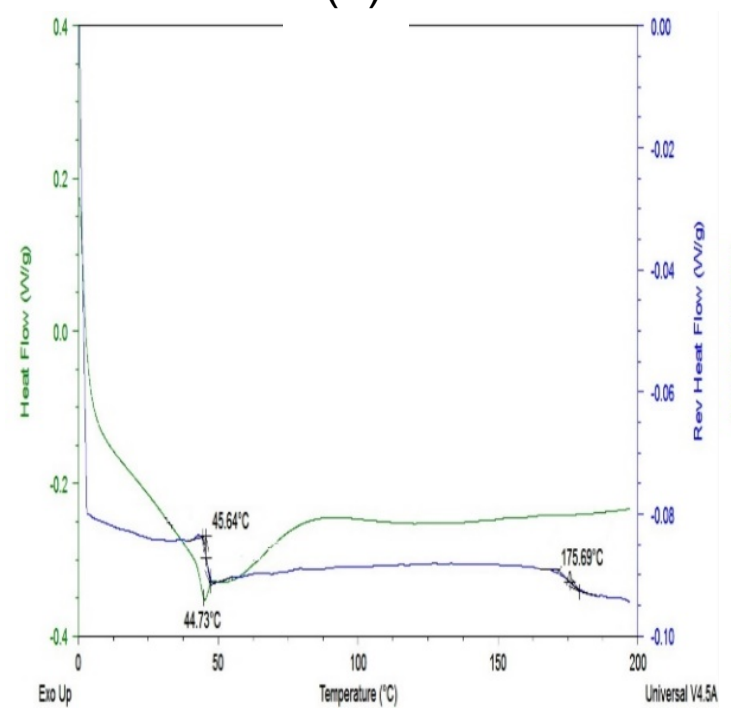

(b)

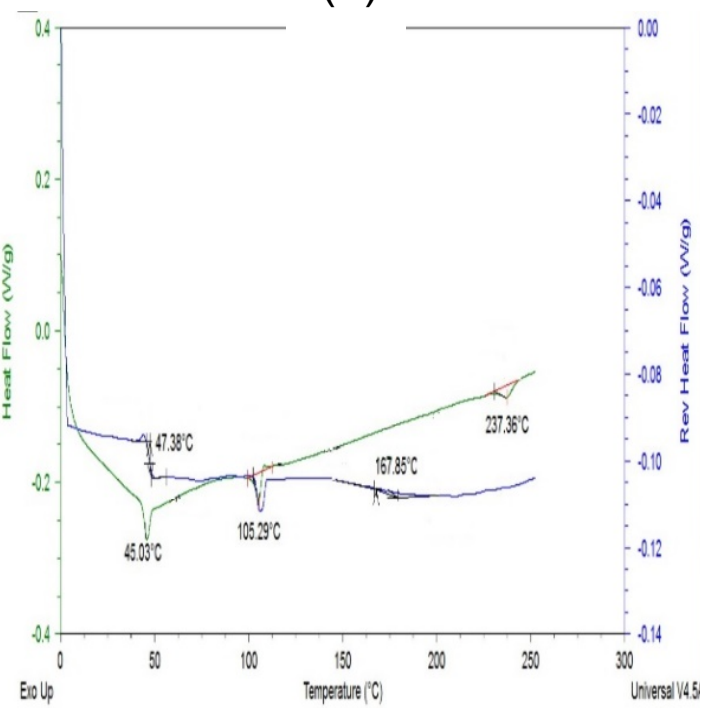


(c)

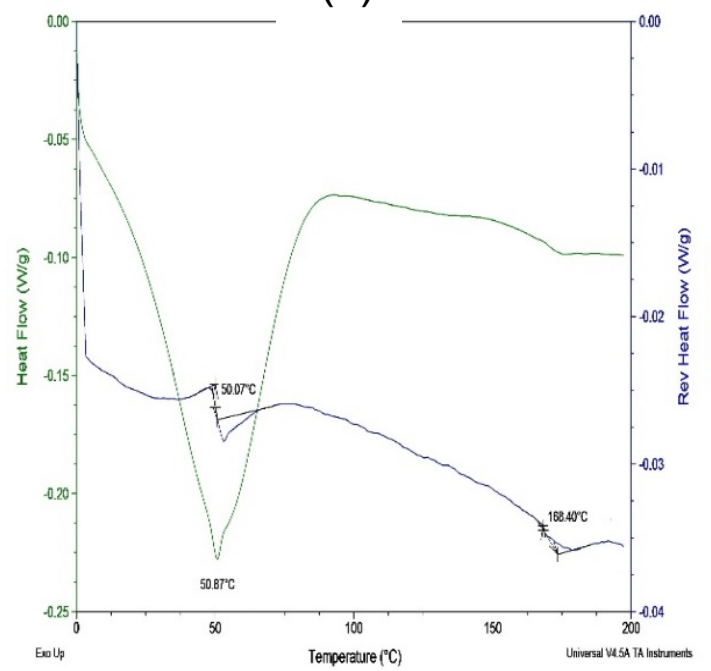

(d)

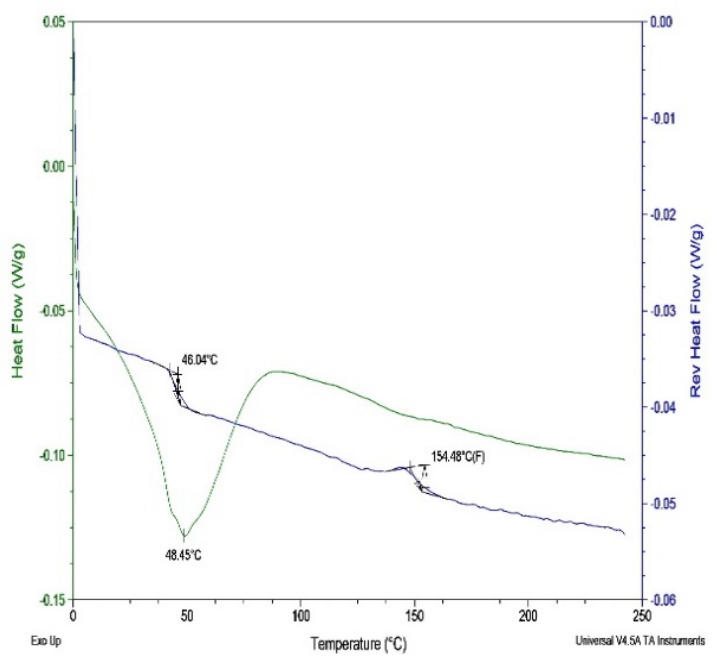

Figure 4: MTDSC traces of (a) blank PLGA and PVP PM, (b) drug-loaded PLGA and PVP PM, (c) blank coaxial fibers and (d) drug-loaded coaxial fibers.

Overall, the thermal studies have indicated that the spinning process may result in some residual solvent remaining in the fibers (an issue that will obviously need to be addressed further down the development process) but also that the drugs do appear to be molecularly dispersed in the polymers (pirfenidone in PLGA and moxifloxacin in PVP).

\subsection{Characterization of the physical state}

The FTIR spectra of the pure polymers and drugs are illustrated in Figure 5a. The PLGA FTIR spectrum exhibited a C $=\mathrm{O}$ stretch at $1746 \mathrm{~cm}^{-1}$ and a C-O-C stretch at $1100 \mathrm{~cm}^{-1}$. These spectra demonstrated carbonyl stretching due to the presence of an ester group. This is in consistent with the PLGA FTIR spectrum of Zhang et al. (2012), Khalil et al. (2013) and Zhu et al. (2015). The characteristic peaks of PVP were seen at $1658 \mathrm{~cm}^{-1}$ ( $\mathrm{C}=\mathrm{O}$ stretching), $1455 \mathrm{~cm}^{-1}(\mathrm{C}-\mathrm{H}$ bending in aliphatic compound) and $1280 \mathrm{~cm}^{-1}$ (C-N stretching in aromatic amine). Due to the hygroscopic nature of PVP, a broad band was observed at $3418 \mathrm{~cm}^{-1}$ indicating the presence of $\mathrm{O}-\mathrm{H}$ stretch. This spectrum is in agreement with Illangakoon et al. (2014), Wang et al. (2015) and Asawahame et al. (2015) FTIR spectra for PVP.

The FTIR spectrum for pirfenidone demonstrated stretching at $3050 \mathrm{~cm}^{-1}$ (aromatic $\mathrm{C}-\mathrm{H}$ stretching), $2929 \mathrm{~cm}^{-1}$ (aliphatic $\mathrm{C}-\mathrm{H}$ stretching), $1671 \mathrm{~cm}^{-1}$ (C=O stretching in tertiary amide), $1622-1454 \mathrm{~cm}^{-1}$, (aromatic $\mathrm{C}=\mathrm{C}$ and pyridine ring stretching) and $1273-1260 \mathrm{~cm}^{-1}$ (C-N stretching), which is similar to the pirfenidone FTIR spectrum of Soni et al. (2018). In addition, sharp bands between $824 \mathrm{~cm}^{-1}$ and $699 \mathrm{~cm}^{-1}$ are present in the fingerprint region that represent substitutions in the benzene ring. The moxifloxacin FTIR spectrum showed some key peaks at $3523-3470 \mathrm{~cm}^{-1}(\mathrm{~N}-\mathrm{H}$ aromatic amine stretching), $2950 \mathrm{~cm}^{-1}$ (aliphatic $\mathrm{C}-\mathrm{H}$ stretching), $1703 \mathrm{~cm}^{-1}$ (carboxylic acid $\mathrm{C}=\mathrm{O}$ stretching), $1621 \mathrm{~cm}^{-1}$ (pyridine ring stretching), $1513 \mathrm{~cm}^{-1}$ (aromatic benzene ring stretching), $1454-1423 \mathrm{~cm}^{-1}$ (carboxylic acid O-H stretching) and $1280-1180 \mathrm{~cm}^{-1}$ (aromatic amine C-N stretching). In addition, characteristic peaks were observed at the fingerprint region at about $780-720 \mathrm{~cm}^{-1}$, which represent the 
stretch of substitutions in the benzene ring. This spectrum is in agreement with those reported by Sabitha et al. (2012) and Mudgil and Pawar (2013).

The FTIR spectra for the blank and drug-loaded coaxial fibers and their PMs suggested the presence of both polymers. This is due to the detection of the characteristic peaks at $1746 \mathrm{~cm}^{-1}\left(\mathrm{C}=\mathrm{O}\right.$ stretch) for PLGA and at $3418 \mathrm{~cm}^{-1}(\mathrm{O}-\mathrm{H}$ stretching) and at $1658 \mathrm{~cm}^{-1},(\mathrm{C}=\mathrm{O}$ stretching) for PVP, as shown in Figure $5 \mathrm{~b}$. In addition, the pirfenidone peak at around $1530 \mathrm{~cm}^{-1}$ and the moxifloxacin peak at approximately $770 \mathrm{~cm}^{-1}$ can be observed in the drug-loaded PMs but not in the blank PMs and fibers (Figure 5b). This indicates the presence of these drugs within these formulations, also suggesting the absence of chemical interactions between the drug and the polymer due to the electrospinning process. Numerous studies, such as Zhang et al. (2012) on captopril loaded PLGA, PCL or PLA nanofibers, Khalil et al. (2013) on silver loaded PLGA nanofibers, Wang et al. (2015) on curcumin loaded PVP nanofibers and Zhu et al. (2015) on flurbiprofen axetil loaded PLGA/PVP coaxial fibers, have shown a consistent finding of no interaction between the loaded drug and the polymer after the fabrication process.

(a)

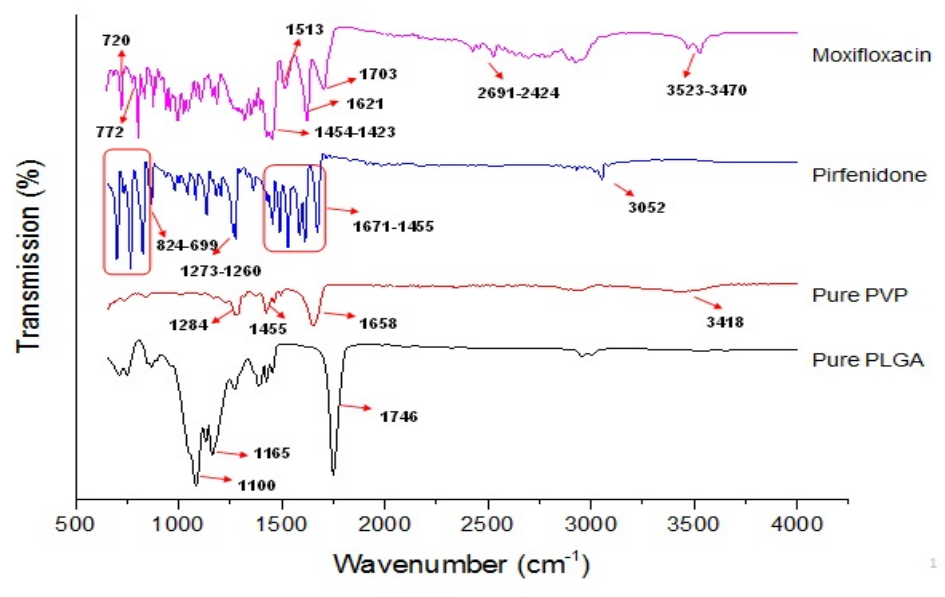


(b)

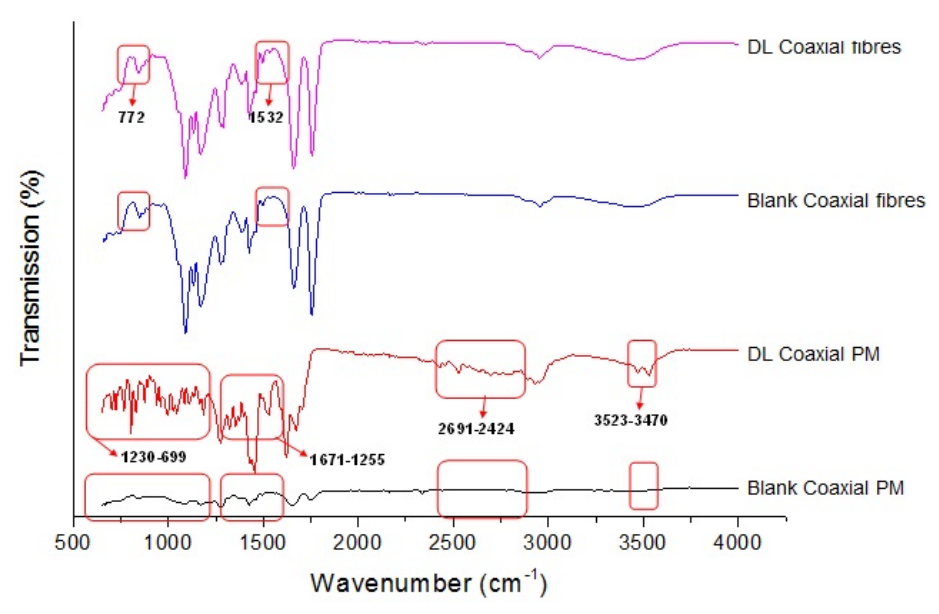

Figure 5: FTIR spectra of (a) the pure PLGA, PVP, pirfenidone and moxifloxacin, showing each material distinctive peaks and (b) the blank and drug-loaded coaxial fibers and their PMs showing pirfenidone and moxifloxacin distinctive peaks in the drug-loaded coaxial fibers and its PM.

XRD diffraction patterns for the pure polymers and drugs are presented in Figure 6 (a). PLGA and PVP are amorphous polymers and hence will not be expected to show any crystalline peaks. Mudgil and Pawar (2013) and Asawahame et al. (2015) demonstrated broad halos in the XRD patterns for PLGA and PVP polymers, respectively. However, for the pure pirfenidone and moxifloxacin, a very clear characteristic reflection is observed in their XRD pattern. Distinct peaks at $8.90^{\circ}$, $14.94^{\circ}, 19.04^{\circ}, 23.08^{\circ}, 24.80^{\circ}$ and $27.60^{\circ}$ were observed in pirfenidone diffractogram, which is similar to the findings of Kashikar et al. (2014) and Soni et al. (2018) pirfenidone diffractograms. The moxifloxacin XRD pattern showed diffraction peaks at $5.90^{\circ}, 8.62^{\circ}, 11.81^{\circ}, 14.48^{\circ}, 15.58^{\circ}, 17.34^{\circ}, 24.18^{\circ}, 26.60^{\circ}, 27.46^{\circ}$ and $31.54^{\circ}$, which are in agreement with the moxifloxacin XRD pattern of Mudgil and Pawar (2013). Both pirfenidone and moxifloxacin peaks appeared at diffraction angles of $18.45^{\circ}, 20.99^{\circ}, 23.21^{\circ}, 27.27^{\circ}, 28.45^{\circ}$ and $32.37^{\circ}$ for the drug-loaded coaxial PM, which were lacking from the blank PMs, as can be seen in Figure 6 (b). However, the blank and drug-loaded coaxial fibers (Figure 6 (b)) exhibited broad halos that were due to the molecular dispersion of both drugs in the coaxial fibers, suggesting that the electrospinning process did not develop a crystalline structure of these fibers. This is supported by several studies such as Zhang et al., 2012, Illangakoon et al., 2014, Wang et al., 2015 and Asawahame et al., 2015. These data are also in agreement with the findings of the MTDSC study which also predicted the drug to be molecularly dispersed in the fibers. 
(a)

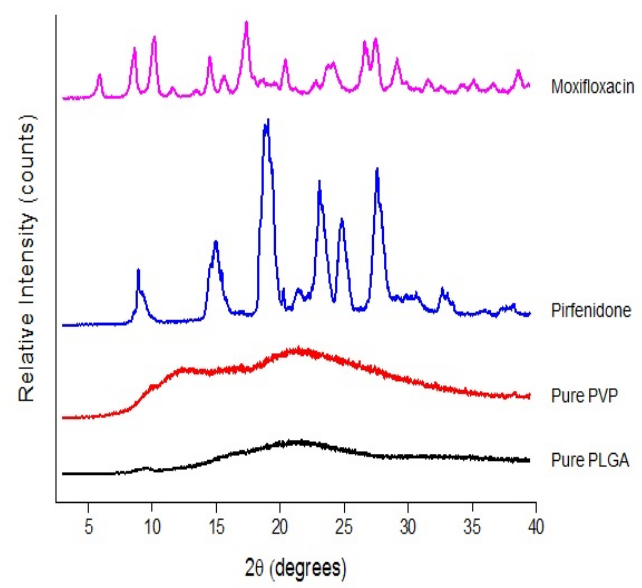

(b)

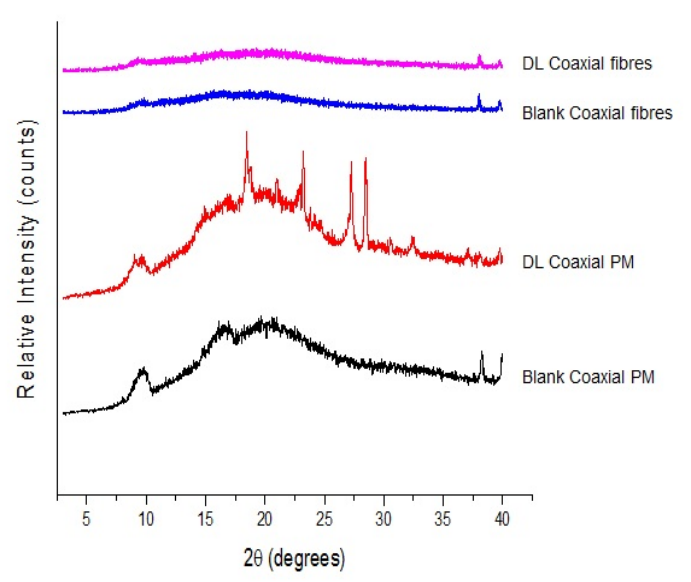

Figure 6: XRD patterns of (a) pure PLGA, PVP, pirfenidone and moxifloxacin, showing that both drugs are in the crystalline form (distinct peaks) while both polymers are in the amorphous form (broad halos) and (b) blank and drug-loaded coaxial fibers and PM showing that the blank and drug-loaded coaxial fibers and the blank PM are in the amorphous form (broad halos), while there are distinct peaks in the drug-loaded PM.

\subsection{Swelling and weight loss studies}

The swelling test was performed to assess the penetration of PBS into the polymer matrix, facilitating drug release and/or fiber erosion. Figure 7 indicates a maximum in the swelling (324 $\pm 14 \%$ ) after 2 hours contact with PBS, after which the drug-loaded fibers gradually decreased in weight. After 24 hours, the fibers exhibited a swelling \% of $216 \pm 18 \%$, indicating the degradation of the PVP layer. Sun et al. (2006), in their water uptake study on PLA/PVP coaxial fibers, showed greater water sorption than the PLA monoaxial fibers due to the presence of the hydrophilic polymer PVP in the coaxial system. The data presented here therefore indicate firstly that considerable swelling occurs in the system, despite the presence of the largely hydrophobic outer shell of PLGA, and secondly that there is evidence for erosion after two hours.

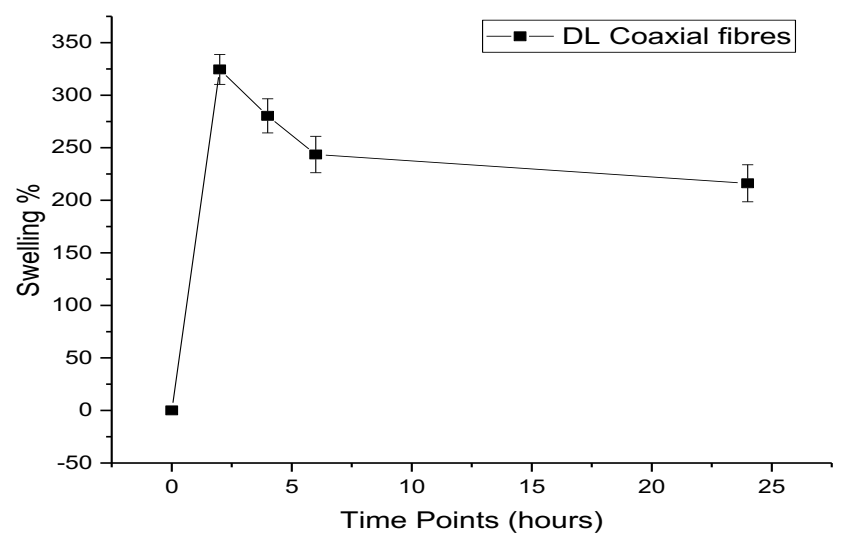

Figure 7: Swelling behaviour of the drug-loaded coaxial fibers showing their swelling $\%$ over time in the release medium. 
The weight loss of the fibers was measured after 24 hours incubation in PBS. The weight loss of the drug-loaded coaxial was $38 \% \pm 2 \%$. This may be attributed to a combination of PLGA erosion, PVP dissolution and drug loss, although it is not possible to delineate the contributions from these from the available data. Nevertheless, it is clear that there is a substantial loss of material from the fibers, including from the polymer matrix, during the dissolution process.

\subsection{Drug loading, entrapment efficiency and drug release}

For measuring the DL, EE and yield of pirfenidone and moxifloxacin from the drugloaded coaxial fibers, two HPLC methods were developed to separate both drugs in ACTN (for DL and EE measurements) and PBS (pH 7.4, for the release measurement). The details of the assay development are presented in the supplementary section.

Owing to the similar flow rate for both the core and the shell $(0.5 \mathrm{~mL} / \mathrm{h})$ in the preparation of the coaxial fibers, the DL of pirfenidone (42 $\pm 1 \mu \mathrm{g} / \mathrm{mg}$ ) was almost double the $\mathrm{DL}$ of moxifloxacin $(23 \pm 1 \mu \mathrm{g} / \mathrm{mg})$. This is due to the initial twofold concentration difference of pirfenidone $(2 \% \mathrm{w} / \mathrm{v})$ to moxifloxacin $(1 \% \mathrm{w} / \mathrm{v})$. The EE of pirfenidone and moxifloxacin was $80 \pm 2 \%$ and $87.5 \pm 3 \%$, respectively. The overall yield (i.e. the weight of fibers harvested as a function of the theoretical) of the prepared drug-loaded fibers was $64 \pm 5 \%$.

The drug-loaded coaxial system showed a rapid release of pirfenidone (39\%) after 30 minutes then a complete release after 24 hours, as shown in Figure 8 . However, moxifloxacin also demonstrated a rapid release of $60 \%$ after 30 minutes followed by a $10 \%$ increment $(70 \%$ cumulative release) after 2 hours after which the concentration remained constant for the 24 hour experimental period. In addition, the EE\% of the fibrous mat was measured at the end of the release study (24 hours) in order to estimate the amount of the entrapped moxifloxacin. The measured EE\% was shown to be $24.5 \% \pm 1.5 \%$ that corresponded to the remaining amount of moxifloxacin. This indicated that a considerable proportion of the moxifloxacin was retained in the nanofibers. The fibres were smooth but highly entangled in appearance after the dissolution period (Figure 9), reflecting the solvent ingress and associated plasticization. 


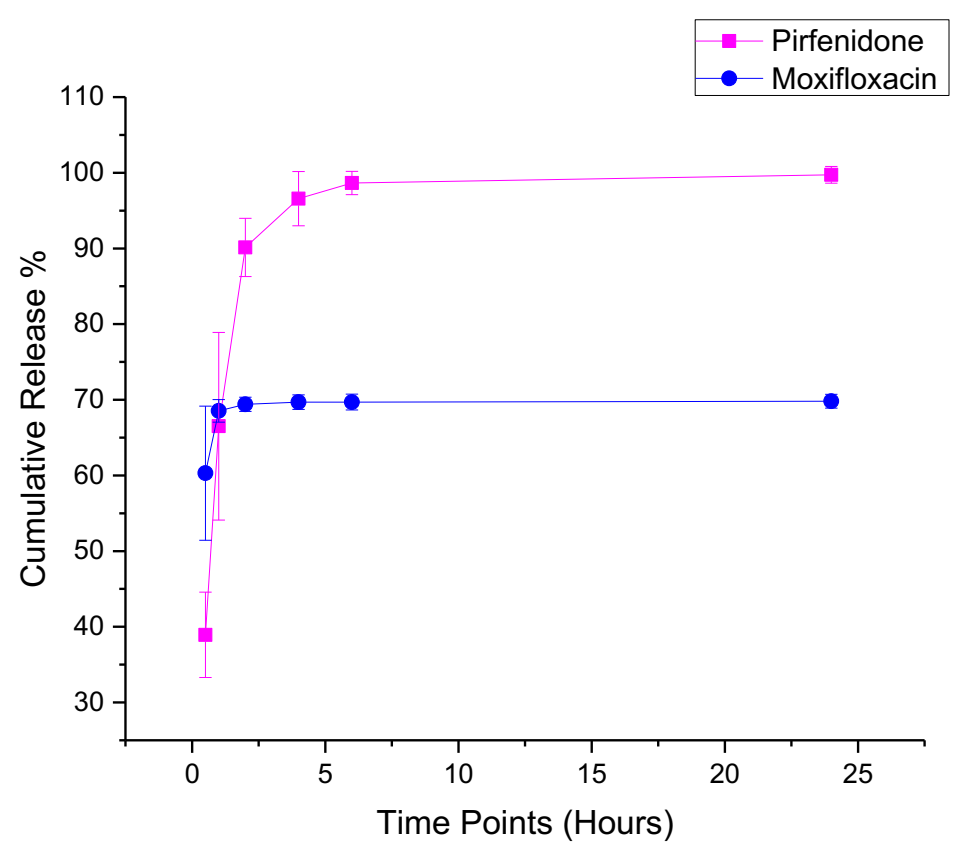

Figure 8: The release profile of the drug-loaded coaxial fibers. The drug-loaded fibers showed a burst release of pirfenidone after 30 minutes followed by a complete release after 24 hours compared to a burst release of moxifloxacin after 30 minutes followed by a plateau release to at least 24 hours.
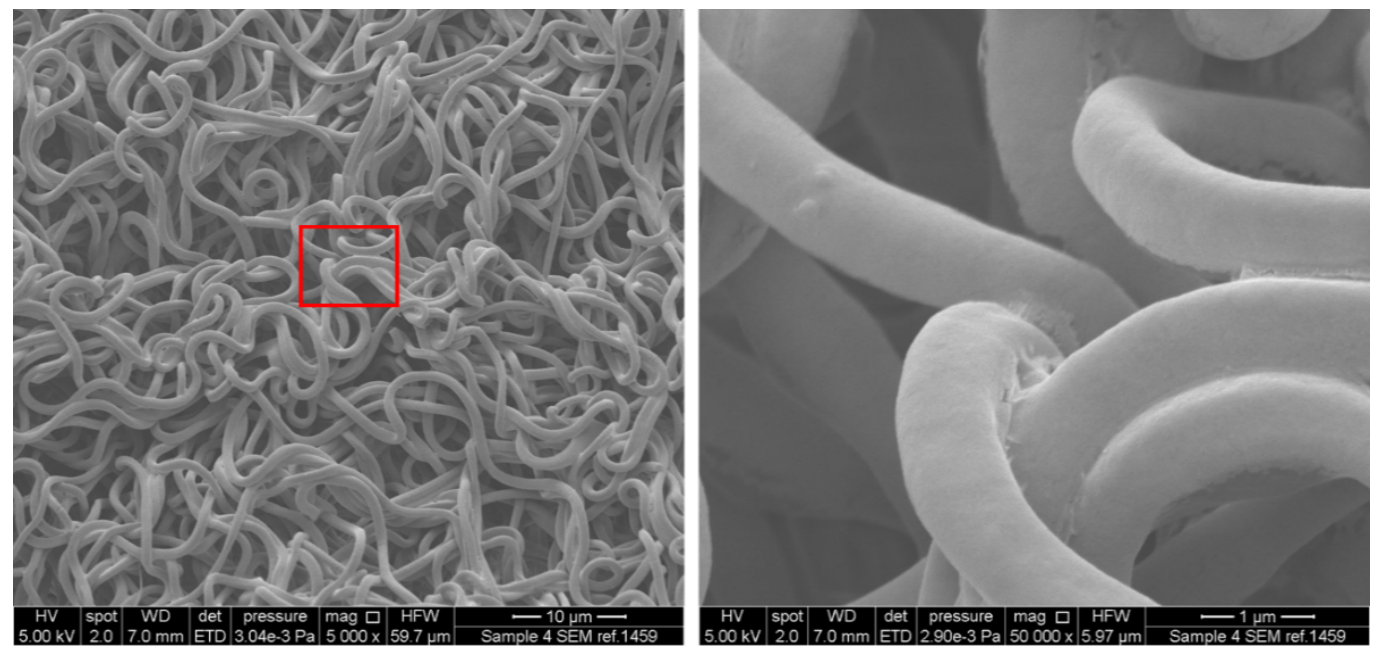

Figure 9: SEM image of the drug-loaded coaxial fibers after 24 hours release. Fibers are entangled and the surfaces smooth.

This complex release profile is consistent with previous studies on coaxial fibers. For example, Zhu et al. (2015) fabricated a PLGA/PVP coaxial system loaded with flurbiprofen axetil in the core, which has an initial burst release (40\%) after 6 hours followed by a sustained release $(70 \%)$ after 204 hours. In addition, Han and Steckl (2013) demonstrated approximately 50\% release of the core drug after 24 hours from PCL/PVP immiscible coaxial system (PCL was dissolved in chloroform and PVP was dissolved in water). However, both the core and the shell drugs were released 
completely after 24 hours and 2 hours, respectively, from the miscible coaxial system (PCL was dissolved in trifluoroethanol and PVP was dissolved in water). A study by He et al. (2018) reported a sustained release of PLGA-metronidazole/PVP-naringin coaxial system, with the shell drug (metronidazole) exhibited a faster release profile than the core drug (naringin). A complete release of both drugs was seen after 5 days. Han and Steckl (2013) also showed an accelerated release of both the core and the shell drugs from PCL/PVP systems.

The release of the pirfenidone is consistent with reasonable expectations when using a shell composed of a relatively hydrophilic grade of PLGA (50:50) in that the polymer clearly swells in the presence of buffer and the drug is released relatively rapidly through a combination of diffusion, erosion and surface release; the high surface area/volume ratio and indeed high absolute surface area of the fibers undoubtedly contributes to this effect. The timescale of release (circa 1-2 hours) is encouraging as this may be easily tailored by, for example, the use of more hydrophobic grades so as to allow release over several hours, thereby allowing once daily dosing.

The more problematic outcome, from both a mechanistic and performance viewpoint, is the release of the moxifloxacin, whereby they were both a rapid initial release and a significant retention within the fibers. We suggest that the initial rapid release, which is surprising given the encapsulation of the PVP in the PLGA shell, reflects the penetration of water into the core via the swollen PLGA; the PVP and the drug may be released via a number of mechanisms, including (drug) diffusion across the swollen PLGA, hydrodynamic extrusion out of the fiber ends or through flaws in the fiber structures. Indeed, the overall weight loss suggests that a significant quantity of PVP is being lost from the system. It may be hypothesised that the PVP will eventually (i.e. after the initial release) equilibrate with the environment and become effectively inert, resulting in the drug remaining entrapped within the fiber core. Further work is ongoing to investigate this mechanism. Overall, there does need to be further work to both tailor the release rate and improve the efficiency of overall release, although again the time period of release is encouraging and similarly may well be easily modified by use of a more hydrophobic PLGA.

A further aspect that may be manipulated is the thickness of the two layers. The TEM image in Figure 2 showed that the thickness ratio between the layers of the coaxial fibers is almost double, with the inner layer estimated as $167 \mathrm{~nm}$ and the outer layer $384 \mathrm{~nm}$. This factor has been explored by Ball et al. (2016) who studied fibers with a hydrophobic polymer ethyl cellulose outer layer and PVP inner layer. They reported that by increasing the thickness of the outer layer by enhancing the shell-to-core volume ratio from 0.5 to 4 , the release of maraviroc from the core was extended from 1 to 5 days. Another study by Sriyanti et al. (2018) demonstrated that the release of a-mangostin from PVP nanofibers was faster from the smaller fiber diameter (387 nm) due to higher rate of release medium absorbed compared to the larger diameter fibers $(468 \mathrm{~nm})$. This allowed the polymer chain to relax which led to fibers swelling. This can further dissolve the drug rapidly (by the penetrating solvent) allowing its diffusion out of the polymer matrix. 


\section{Conclusion}

Pirfenidone-PLGA/moxifloxacin-PVP coaxial fibers were successfully prepared using coaxial electrospinning technique. The SEM images illustrate the production of unbeaded, non-porous and smooth fiber's surface, while the TEM finding has shown the presence of two distinctive layer suggesting the successful coaxial fabrication of the drug-loaded fibers. Fluorescence microscopy indicated the encapsulation of moxifloxacin, which was located in the core of the coaxial fibers. The Y\% of the drugloaded coaxial fibers was $64 \pm 5 \%$, with an EE\% of moxifloxacin and pirfenidone 87.5 $\pm 3 \%$ and $80 \pm 2 \%$, respectively. Nevertheless, due to the similar flow rate $(0.5 \mathrm{~mL} / \mathrm{h})$ that was used to prepare the coaxial fibers, the DL of both drugs (42 $\pm 1 \mu \mathrm{g} / \mathrm{mg}$ for pirfenidone and $23 \pm 1 \mu \mathrm{g} / \mathrm{mg}$ for moxifloxacin) were accordingly to the initial concentration ratio (2:1). DSC and XRD confirmed the molecular dispersion of both drugs in the fibers.

The release profile for the drug-loaded coaxial fibers demonstrated a complete release of pirfenidone after circa 2 hours, while $60 \%$ of moxifloxacin was release after 30 minutes with the remaining $40 \%$ entrapped in the core of these fibers. We suggest that even though there is further work required to tailor the release profile, these initial results are highly encouraging as they demonstrated that sustained release is possible over a period of hours and that effective entrapment in the two compartments is possible. As the purpose of the study was to provide a basis for the treatment of corneal abrasion via a single daily dose of the drug-loaded fiber system, the release profile was sufficiently satisfactory to take this formulation forward into in vitro and in vivo studies in order to evaluate the bioactivity of the loaded drugs.

\section{Acknowledgement}

We would like to thank King Abdulaziz City for Science and Technology for their financial support of ET

\section{References}

Aguilar, Z.P., 2013. Chapter 5 - Targeted Drug Delivery. Nanomaterials for Medical Applications, pp.181-234.

Asawahame, C., Sutjarittangtham, K., Eitssayeam, S., Tragoolpua, Y., Sirithunyalug, B. and Sirithunyalug, J., 2015. Formation of orally fast dissolving fibers containing propolis by electrospinning technique. Chiang Mai Journal of Science, 42, pp.469480.

Ball, C., Chou, S.F., Jiang, Y. and Woodrow, K.A., 2016. Coaxially electrospun fiberbased microbicides facilitate broadly tunable release of maraviroc. Materials Science and Engineering: C, 63, pp.117-124.

Bourcier, T., Thomas, F., Borderie, V., Chaumeil, C. and Laroche, L., 2003. Bacterial keratitis: predisposing factors, clinical and microbiological review of 300 cases. British Journal of Ophthalmology, 87(7), pp.834-838.

Bukowiecki, A., Hos, D., Cursiefen, C. and Eming, S., 2017. Wound-healing studies in cornea and skin: parallels, differences and opportunities. International journal of molecular sciences, 18(6), pp.1257-1281. 
Chakraborty, S., Liao, I.C., Adler, A. and Leong, K.W., 2009. Electrohydrodynamics: a facile technique to fabricate drug delivery systems. Advanced drug delivery reviews, 61(12), pp.1043-1054.

Chan, K.P., Chu, K.O., Lai, W.W.K., Choy, K.W., Wang, C.C., Lam, D.S.C. and Pang, C.P., 2006. Determination of ofloxacin and moxifloxacin and their penetration in human aqueous and vitreous humor by using high-performance liquid chromatography fluorescence detection. Analytical biochemistry, 353(1), pp.30-36.

Cheng, K.H., Leung, S.L., Hoekman, H.W., Beekhuis, W.H., Mulder, P.G., Geerards, A.J. and Kijlstra, A., 1999. Incidence of contact-lens-associated microbial keratitis and its related morbidity. The Lancet, 354(9174), pp.181-185.

Coleman, N.J. and Craig, D.Q., 1996. Modulated temperature differential scanning calorimetry: a novel approach to pharmaceutical thermal analysis. International journal of pharmaceutics, 135(1-2), pp.13-29.

Dewani, A.P., Barik, B.B., Kanungo, S.K., Wattyani, B.R. and Chandewar, A.V., 2011. Development and validation of RP-HPLC method for the determination of moxifloxacin in presence of its degradation products. American-Eurasian Journal of Scientific Research, 6(4), pp.192-200.

Du Bois, R.M., 2010. Strategies for treating idiopathic pulmonary fibrosis. Nature reviews Drug discovery, 9(2), pp.129-140.

European Medicines Agency (EMA). European Public Assessment Report - Esbriet. EMA website, viewed in 15 November 2015, $<$ https://www.ema.europa.eu/en/medicines/human/EPAR/esbriet>.

Garg, T., Rath, G. and Goyal, A.K., 2015. Biomaterials-based nanofiber scaffold: targeted and controlled carrier for cell and drug delivery. Journal of drug targeting, 23(3), pp.202-221

Han, D. and Steckl, A.J., 2013. Triaxial electrospun nanofiber membranes for controlled dual release of functional molecules. ACS applied materials \& interfaces, 5(16), pp.8241-8245.

He, P., Zhong, Q., Ge, Y., Guo, Z., Tian, J., Zhou, Y., Ding, S., Li, H. and Zhou, C., 2018. Dual drug loaded coaxial electrospun PLGA/PVP fiber for guided tissue regeneration under control of infection. Materials Science and Engineering: C, 90, pp.549-556.

Illangakoon, U.E., Gill, H., Shearman, G.C., Parhizkar, M., Mahalingam, S., Chatterton, N.P. and Williams, G.R., 2014. Fast dissolving paracetamol/caffeine nanofibers prepared by electrospinning. International journal of pharmaceutics, 477(1-2), pp.369-379.

Iyer, S.N., Gurujeyalakshmi, G. and Giri, S.N., 1999. Effects of pirfenidone on transforming growth factor- $\beta$ gene expression at the transcriptional level in bleomycin hamster model of lung fibrosis. Journal of Pharmacology and Experimental Therapeutics, 291(1), pp.367-373.

Jamarani, R., Erythropel, H., Nicell, J., Leask, R. and Marić, M., 2018. How green is your plasticizer?. Polymers, 10(8), pp.834-851. 
Jeng, B.H. and McLeod, S.D., 2003. Microbial keratitis. Br. J. Ophthalmol. 87, pp. 805-806.

Jung, K.I., Choi, J.S., Kim, H.K. and Shin, S.Y., 2012. Effects of an anti-transforming growth factor- $\beta$ agent (pirfenidone) on strabismus surgery in rabbits. Current eye research, 37(9), pp.770-776.

Kashikar, V., Dhole, S., Kandekar, U. and Khose, P., 2014. Study of mucoadhesive microsphere of pirfenidone for nasal drug delivery. Asian Journal of Pharmaceutics (AJP), 8(1), pp. 46-53.

Keay, L., Edwards, K., Naduvilath, T., Taylor, H.R., Snibson, G.R., Forde, K. and Stapleton, F., 2006. Microbial keratitis: predisposing factors and morbidity. Ophthalmology, 113(1), pp.109-116.

Keay, L., Edwards, K., Naduvilath, T., Forde, K. and Stapleton, F., 2006. Factors affecting the morbidity of contact lens-related microbial keratitis: A population study. Investigative ophthalmology \& visual science, 47(10), pp.4302-4308.

Khalil, K.A., Fouad, H., Elsarnagawy, T. and Almajhdi, F.N., 2013. Preparation and characterization of electrospun PLGA/silver composite nanofibers for biomedical applications. Int J Electrochem Sci, 8(3), pp.3483-3493.

Kinoshita, S., Adachi, W., Sotozono, C., Nishida, K., Yokoi, N., Quantock, A.J. and Okubo, K., 2001. Characteristics of the human ocular surface epithelium. Progress in retinal and eye research, 20(5), pp.639-673.

Lee, K., Young Lee, S., Park, S.Y. and Yang, H., 2014. Antifibrotic effect of pirfenidone on human pterygium fibroblasts. Current eye research, 39(7), pp.680-685.

Lin, X., Yu, M., Wu, K., Yuan, H. and Zhong, H., 2009. Effects of pirfenidone on proliferation, migration, and collagen contraction of human Tenon's fibroblasts in vitro. Investigative ophthalmology \& visual science, 50(8), pp.3763-3770.

Ljubimov, A.V. and Saghizadeh, M., 2015. Progress in corneal wound healing. Progress in retinal and eye research, 49, pp.17-45.

Miller, D., 2008. Review of moxifloxacin hydrochloride ophthalmic solution in the treatment of bacterial eye infections. Clinical ophthalmology (Auckland, NZ), 2(1), pp.77-91.

Mudgil, M. and Pawar, P., 2013. Preparation and in vitrolex vivo evaluation of moxifloxacin-loaded PLGA nanosuspensions for ophthalmic application. Scientia pharmaceutica, 81(2), pp.591-606.

Murugan, R. and Ramakrishna, S., 2007. Design strategies of tissue engineering scaffolds with controlled fiber orientation. Tissue engineering, 13(8), pp.1845-1866

Ong, H.S. and Corbett, M.C., 2015. Corneal infections in the 21st century. Postgraduate medical journal, 91(1080), pp.565-571.

Parmar, V.K., Desai, S.B. and Vaja, T., 2014. RP-HPLC and UV spectrophotometric methods for estimation of pirfenidone in pharmaceutical formulations. Indian journal of pharmaceutical sciences, 76(3), pp.225-229.

Raghu, G., Collard, H.R., Egan, J.J., Martinez, F.J., Behr, J., Brown, K.K., Colby, T.V., Cordier, J.F., Flaherty, K.R., Lasky, J.A. and Lynch, D.A., 2011. An official 
ATS/ERS/JRS/ALAT statement: idiopathic pulmonary fibrosis: evidence-based guidelines for diagnosis and management. American journal of respiratory and critical care medicine, 183(6), pp.788-824.

Ravisankar, P., Rani, K.A., Devadasu, C., Rao, G.D., 2014. A noval validated RPHPLC method for determination of Pirfenidone in pharmaceutical dosage forms. Der Pharmacia lettre. 6(5), pp.19-29.

Romano, L., Camposeo, A., Manco, R., Moffa, M. and Pisignano, D., 2016. CoreShell Electrospun Fibers Encapsulating Chromophores or Luminescent Proteins for Microscopically Controlled Molecular Release. Molecular pharmaceutics, 13(3), pp.729-736.

Sabitha, K., Sajeeth, C.I. and Santhi, K., 2012. Chitosan nanoparticles: a novel vehicle for the enhanced ocular delivery of moxifloxacin $\mathrm{HCl}$. Research Journal of Pharmaceutical, Biological and Chemical Sciences, 3(2), pp.534-548.

Salomao, M.Q., Chaurasia, S.S., Sinha-Roy, A., Ambrósio, R., Esposito, A., Sepulveda, R., Agrawal, V. and Wilson, S.E., 2011. Corneal wound healing after ultraviolet-A/riboflavin collagen cross-linking: a rabbit study. Journal of Refractive Surgery, 27(6), pp.401-407.

Shahid, S.M. and Harrison, N., 2013. Corneal abrasion: assessment and management. InnovAiT, 6(9), pp.551-554.

Soni, S.R., Bhunia, B.K., Kumari, N., Dan, S., Mukherjee, S., Mandal, B.B. and Ghosh, A., 2018. Therapeutically Effective Controlled Release Formulation of Pirfenidone from Nontoxic Biocompatible Carboxymethyl Pullulan-Poly (vinyl alcohol) Interpenetrating Polymer Networks. ACS omega, 3(9), pp.11993-12009.

Sriyanti, I., Edikresnha, D., Rahma, A., Munir, M.M., Rachmawati, H. and Khairurrijal, K., 2018. Mangosteen pericarp extract embedded in electrospun PVP nanofiber mats: physicochemical properties and release mechanism of a-mangostin. International journal of nanomedicine, 13, pp.4927-4941.

Stapleton, F. and Carnt, N., 2012. Contact lens-related microbial keratitis: how have epidemiology and genetics helped us with pathogenesis and prophylaxis. Eye, 26(2), pp.185-193.

Sun, B., Duan, B. and Yuan, X., 2006. Preparation of core/shell PVP/PLA ultrafine fibers by coaxial electrospinning. Journal of Applied Polymer Science, 102(1), pp.3945.

Sun, G., Lin, X., Zhong, H., Yang, Y., Qiu, X., Ye, C., Wu, K. and Yu, M., 2011. Pharmacokinetics of pirfenidone after topical administration in rabbit eye. Molecular vision, 17, pp.2191-2196.

Tiwari, S.K., Tzezana, R., Zussman, E. and Venkatraman, S.S., 2010. Optimizing partition-controlled drug release from electrospun core-shell fibers. International journal of pharmaceutics, 392(1-2), pp.209-217.

Wang, C., Ma, C., Wu, Z., Liang, H., Yan, P., Song, J., Ma, N. and Zhao, Q., 2015. Enhanced bioavailability and anticancer effect of curcumin-loaded electrospun nanofiber: in vitro and in vivo study. Nanoscale research letters, 10(1), pp.439-448. 
Wang, T., Jang, W.H., Lee, S., Yoon, C.J., Lee, J.H., Kim, B., Hwang, S., Hong, C.P., Yoon, Y., Lee, G. and Le, V.H., 2016. Moxifloxacin: Clinically compatible contrast agent for multiphoton imaging. Scientific reports, 6, pp.27142-27149.

Watson, D.G., 2005. Pharmaceutical Analysis, A Textbook for Pharmacy Students and Pharmaceutical Chemists, 2nd ed. Churchill Livingstone Elsevier: London pp.221-233.

Williams, G.R., Raimi-Abraham, B.T. and Luo, C.J., 2018. Electrospinning fundamentals. In: Nanofibres in drug delivery, 1st ed. UCL Press: London pp.24-55.

Wilson, S.E., Liu, J.J. and Mohan, R.R., 1999. Stromal-epithelial interactions in the cornea. Progress in retinal and eye research, 18(3), pp.293-309.

Wise, R., 1999. A review of the clinical pharmacology of moxifloxacin, a new 8methoxyquinolone, and its potential relation to therapeutic efficacy. Clinical drug investigation, 17(5), pp.365-387.

Wong, T., Ormonde, S., Gamble, G. and McGhee, C.N.J., 2003. Severe infective keratitis leading to hospital admission in New Zealand. British Journal of Ophthalmology, 87(9), pp.1103-1108

Zhang, Y.Z., Wang, X., Feng, Y., Li, J., Lim, C.T. and Ramakrishna, S., 2006. Coaxial electrospinning of (fluorescein isothiocyanate-conjugated bovine serum albumin)encapsulated poly ( $\varepsilon$-caprolactone) nanofibers for sustained release. Biomacromolecules, 7(4), pp.1049-1057.

Zhang, Y., Huo, M., Zhou, J., Zou, A., Li, W., Yao, C. and Xie, S., 2010. DDSolver: an add-in program for modeling and comparison of drug dissolution profiles. The AAPS journal, 12(3), pp.263-271.

Zhang, H., Lou, S., Williams, G.R., Branford-White, C., Nie, H., Quan, J. and Zhu, L.M., 2012. A systematic study of captopril-loaded polyester fiber mats prepared by electrospinning. International journal of pharmaceutics, 439(1-2), pp.100-108.

Zhi-Hua, Z., Yun-Ze, L., Hong-Xin, Y., Bin, S., Jie, Z., Hong-Di, Z., Xin-Ming, J. and Chang-Zhi, G., 2012. Electrospun fluorescein/polymer composite nanofibers and their photoluminescent properties. Chinese Physics B, 21(9), pp.097805(1)-097805(6).

Zhong, H., Sun, G., Lin, X., Wu, K. and Yu, M., 2011. Evaluation of pirfenidone as a new postoperative antiscarring agent in experimental glaucoma surgery. Investigative ophthalmology \& visual science, 52(6), pp.3136-3142.

Zhu, T., Chen, S., Li, W., Lou, J. and Wang, J., 2015. Flurbiprofen axetil loaded coaxial electrospun poly (vinyl pyrrolidone)-nanopoly (lactic-co-glycolic acid) core-shell composite nanofibers: Preparation, characterisation, and anti-adhesion activity. Journal of Applied Polymer Science, 132(22), 41982(1)-41982(10). 